\title{
Brane worlds, string cosmology, and AdS/CFT
}

\author{
Luis Anchordoqui, ${ }^{1, *}$ José Edelstein, ${ }^{2, \dagger}$ Carlos Nuñez, ${ }^{2, \ddagger}$ Santiago Perez Bergliaffa, ${ }^{3, \S}$ Martin Schvellinger, ${ }^{4, \mid}$ \\ Marta Trobo, ${ }^{5, \uparrow}$ and Fabio Zyserman ${ }^{5, * *}$ \\ ${ }^{1}$ Department of Physics, Northeastern University, Boston, Massachusetts 02115 \\ ${ }^{2}$ Department of Physics, Harvard University, Cambridge, Massachusetts 02138 \\ ${ }^{3}$ Centro Brasileiro de Pesquisas Fisicas, Rua Xavier Sigaud, 150, CEP 22290-180, Rio de Janeiro, Brazil \\ ${ }^{4}$ Center for Theoretical Physics, Massachusetts Institute of Technology, Cambridge, Massachusetts 02139 \\ ${ }^{5}$ Departamento de Física, Universidad Nacional de La Plata, CC67 La Plata (1900), Argentina
}

(Received 25 June 2001; published 26 September 2001)

\begin{abstract}
Using the thin-shell formalism we discuss the motion of domain walls in de Sitter and anti-de Sitter (AdS) time-dependent bulks. This motion results in a dynamics for the brane scale factor. We show that in the case of a clean brane the scale factor describes both singular and nonsingular universes, with phases of contraction and expansion. These phases can be understood as quotients of AdS spacetime by a discrete symmetry group. We discuss this effect in some detail, and suggest how the AdS/CFT correspondence could be applied to obtain a nonperturbative description of brane-world string cosmology.
\end{abstract}

DOI: $10.1103 /$ PhysRevD.64.084027

PACS number(s): 04.50.+h

\section{INTRODUCTION}

The intriguing idea that fundamental interactions could be understood as manifestations of the existence of extra dimensions in our 4-dimensional world can be traced back at least to the work of Kaluza and Klein (KK) [1], with revivals of activity that one generically refers to as KK theories [2]. Over the last two years, a fresh interest in the topic has been rekindled, mainly due to the realization that localization of matter [3] and localization of gravity [4] may drastically change the commonly assumed properties of such models.

From the phenomenological perspective the so-called "brane worlds" provide an economic explanation of the hierarchy between the gravitational and electroweak mass scales. In the canonical example of Arkani-Hamed, Dimopoulos and Dvali (ADD) [5], spacetime is a direct product of an ordinary 4-dimensional manifold ("our universe") and a (flat) spatial $n$-torus of common linear size $r_{c}$ and volume $v_{n}=r_{c}^{n}$. Of course, standard model (SM) fields cannot propagate a large distance in the extra dimensions without conflict with observations. This is avoided by trapping the fields in a thin shell of thickness $\delta \sim M_{s}^{-1}$ [6]. The only particles propagating in the $(4+n)$-dimensional bulk are the $(4+n)$ gravitons. Thus, gravity becomes strong in the entire $(4+n)$-dimensional spacetime at a scale $M_{s} \sim$ a few $\mathrm{TeV}$, which is far below the conventional Planck scale, $M_{\mathrm{pl}}$ $\sim 10^{18} \mathrm{GeV}$. Strictly speaking, the low energy effective 4-dimensional Planck scale $M_{\mathrm{pl}}$ is related to the fundamental scale of gravity $M_{*}$ via Gauss' law $M_{\mathrm{pl}}^{2}=M_{*}^{2+n} v_{n}$. For $n$

\footnotetext{
*Electronic address: doqui@hepmail.physics.neu.edu

†Electronic address: edels@lorentz.harvard.edu

*Electronic address: nunez@lorentz.harvard.edu

${ }^{\S}$ Electronic address: sepb@cbpf.br

"Electronic address: martin@ctpbeaker.mit.edu

"Electronic address: trobo@venus.fisica.unlp.edu.ar

**Electronic address: zyserman@venus.fisica.unlp.edu.ar
}

extra dimensions one finds that $r_{c} \approx 10^{30 / n-19} \mathrm{~m}$, assuming $M_{*} \sim 1 \mathrm{TeV}$ [7]. This relation immediately suggests that $n$ $=1$ is ruled out, because $r_{c} \sim 10^{11} \mathrm{~m}$ and, the gravitational interaction would thus be modified at the scale of our solar system. However, already for $n=2, r_{c} \sim 1 \mathrm{~mm}$ - just the scale where our present day experimental knowledge about gravity ends [8]. Furthermore, one can imagine more general scenarios termed asymmetric compactifications, where, e.g., there are $p$ "small" dimensions with sizes of $\sim 1 / \mathrm{TeV}$ and the effective number of large extra dimensions being $n_{\text {eff }}$ $=n-p$. Here, the expected number of extra dimensions should be 6 or 7 as suggested by string theory [9]. Naturally, there is a strong motivation for immediate phenomenological studies to assess the experimental viability of such a radical departure from previous fundamental particle physics. Leaving aside table-top experiments and astrophysics (which requires that $M_{s}>110 \mathrm{TeV}$ for $n=2$, but only around a few $\mathrm{TeV}$ for $n>2[10]$ ), there are two ways of probing this scenario. Namely, via the KK-graviton emission in scattering processes, or else through the exchange of KK towers of gravitons among SM particles [11]. The search for extradimension footprints in collider data has already started. However, as yet no observational evidence has been found [12].

The ADD scenario is based on the fact that the 4-dimensional coordinates are independent of the coordinates of the extra $n$ dimensions. Giving up this assumption can lead to a number of other interesting models with completely different gravitational behaviors. Perhaps the most compelling model along these lines can be built by considering a 5-dimensional anti-de Sitter (AdS) space with a single 4-dimensional boundary [4]. This boundary is taken to be a $p$-brane with intrinsic tension $\sigma$. In the RandallSundrum (RS) world, there is a bound state of the graviton confined to the brane, as well as a continuum of KK modes. At low energies, the bound state dominates over the KK states to give an inverse square law if the AdS radius is sufficiently small. Therefore, Newton's law is recovered on 
the brane, even with an infinitely large fifth dimension. The number of papers discussing variants of this scenario is already very large [13]. Some key papers are [14]. For a comprehensive review the reader is referred to [15].

The presence of large extra dimensions modifies the Friedmann equation on the brane by the addition of nonlinear terms [16] which yield new cosmological scenarios [17]. Fortunately, a world that undergoes a phase of inflation could be long lived in a universe like our own at low energies [18]. Specifically, brane-world scenarios are good candidates to describe our world as long as the normal rate of expansion has been recovered by the epoch of nucleosynthesis [19]. We remind the reader that the universe has to be re-heated up to a temperature $\mathcal{O}(\mathrm{MeV})$ so as to synthethize light elements, because the entropy produced during a cold inflationary phase redshifts away. Therefore, any significant departure from the standard Friedmann-Robertson-Walker (FRW) scenario could only arise at very high energies. A broader study of these ideas is currently underway (for an incomplete list of references, see [20]). In this regard, we initiate here the analysis of new inflationary brane-worlds that arise from surgically modified evolving spacetimes. The dynamics of the bulk in our framework is originated in the symmetries of the dS and AdS spacetimes, without the need of extra fields in the bulk, as in the models discussed in Ref. [21].

The article is divided in two main parts. In Sec. II we derive the equation of motion of a brane using the thin shell formalism [22], in which the field equations are re-written as junction conditions relating the discontinuity in the brane extrinsic curvature to its vacuum energy. Then, we discuss the evolution of a single brane falling into dS and AdS spaces. As a result of its noninteraction with the environment producing the gravitational field, the brane tension obeys an internal conservation law. Therefore, the motion of the brane can be treated as a closed system, or alternatively as a continuous collection of such branes. The evolution yields a dynamics for the scale factor. We show that in the case of a clean brane (i.e., without matter in the form of stringy excitations) the scale factor describes both singular and nonsingular universes, with phases of contraction and expansion. Section III contains a general discussion (and some speculations) on the applications of our results in the light of the AdS/conformal field theory (CFT) correspondence. Quotients of AdS space by discrete symmetry groups describe dynamical AdS bulks. Indeed, it has been suggested [23] that this is the way in which AdS/CFT correspondence must be formulated in the case of dynamical spacetimes. However, since we consider a $p$-brane instead of the AdS boundary itself, for our purposes, the AdS/CFT correspondence should be considered when gravity is coupled to the conformal theory.

\section{BRANE WORLDS FROM THE CONNECTED SUM OF (A)dS}

\section{A. Field equations}

In spite of the fact that dS space does not seem to be obtainable from stringy backgrounds, there has been a grow- ing interest in dS bulks in recent times [24]. In view of these developments we will discuss the motion of spherical branes in both dynamical dS and AdS bulks. The following discussion will refer mostly to branes of dimension $d=4$, but the relevant equations will be written for arbitrary $d \geqslant 2$.

In order to build the class of geometries of interest, we consider two copies of $(d+1)$-dimensional dS (AdS) spaces $\mathcal{M}_{1}$ and $\mathcal{M}_{2}$ undergoing expansion. Then, we remove from each one identical $d$-dimensional regions $\Omega_{1}$ and $\Omega_{2}$ [25]. One is left with two geodesically incomplete manifolds with boundaries given by the hypersurfaces $\partial \Omega_{1}$ and $\partial \Omega_{2}$. Finally, we identify the boundaries up to homeomorphism $h: \partial \Omega_{1} \rightarrow \partial \Omega_{2}$ [26]. Therefore, the resulting manifold that is defined by the connected sum $\mathcal{M}_{1} \# \mathcal{M}_{2}$, is geodesically complete. The classical action of such a system can be cast in the following form:

$$
\begin{aligned}
S= & \frac{L_{p}^{(3-d)}}{16 \pi} \int_{\mathcal{M}} d^{d+1} x \sqrt{g}(R-2 \Lambda) \\
& +\frac{L_{p}^{(3-d)}}{8 \pi} \int_{\partial \Omega} d^{d} x \sqrt{\gamma} \mathfrak{K}+\sigma \int_{\partial \Omega} d^{d} x \sqrt{\gamma} .
\end{aligned}
$$

The first term is the usual Einstein-Hilbert action with a cosmological constant $\Lambda$. The second term is the GibbonsHawking boundary term, necessary for a well-defined variational problem [27]. In our convention, the extrinsic curvature is defined as $\mathfrak{K}_{M N}=\nabla_{(M} \hat{n}_{N)}$, where $\hat{n}^{M}$ is the outward pointing normal vector to the boundary $\partial \Omega$. ${ }^{1}$ Here $R$ stands for the $(d+1)$-dimensional Ricci scalar in terms of the metric $g_{M N}$, while $\gamma$ is the induced metric on the brane, and $\sigma$ is the brane tension.

For definiteness, the spatial coordinates on $\partial \Omega$ can be taken to be the angular variables $\phi_{i}$, which for a spherically symmetric configuration are always well defined up to an overall rotation. Generically, the line element of each patch can be written as

$$
d s^{2}=-d t^{2}+A^{2}(t)\left[r^{2} d \Omega_{(d-1)}^{2}+\left(1-k r^{2}\right)^{-1} d r^{2}\right],
$$

where $k$ takes the value $1(-1)$ for a closed (open) universe, $d \Omega_{(d-1)}$ is the corresponding metric on the unit $(d-1)$-dimensional sphere, and $t$ is the proper time of a clock carried by any raider of the extra dimension. The homeomorphism $h$ entails a proper matching of the metric across the boundary layer [22]. The required junction conditions are most conveniently derived by introducing Gaussian normal coordinates in the vicinity of the brane,

$$
d s^{2}=(\mathbf{n} \cdot \mathbf{n})^{-1} d \eta^{2}+\gamma_{\mu \nu}\left(\eta, x^{\mu}\right) d x^{\mu} d x^{\nu},
$$

\footnotetext{
${ }^{1}$ Throughout the article capital Latin subscripts run from 1 to $(d$ $+1)$, lower Greek subscripts from 1 to $d$, and lower Latin subscripts from 1 to $(d-1)$. As usual, parentheses denote symmetrization; $\nabla$ is the $(d+1)$-dimensional covariant derivative. We adopt geometrodynamic units so that $G \equiv 1, c \equiv 1$ and $\hbar \equiv L_{p}^{2} \equiv M_{p}^{2}$, where $L_{p}$ and $M_{p}$ are the Planck length and Planck mass, respectively.
} 
where $\mathbf{n}=\partial_{\eta},(\mathbf{n} \cdot \mathbf{n})=1,(-1)$ if $\partial \Omega$ is space-like (timelike). The coordinate $\eta$ parametrizes the proper distance perpendicularly measured from $\partial \Omega$. Associating negative values of $\eta$ to one side of the shell and positive values to the other side, the energy momentum tensor $T^{M N}$ can be written as

$$
\begin{aligned}
T^{M N}(x)= & \sigma \eta^{\mu \nu} \delta_{\mu}{ }^{M} \delta_{\nu}{ }^{N} \delta(\eta)+\frac{3 \Lambda L_{p}^{(3-d)}}{2 \pi d(d-1)} \\
& \times g^{M N}[\Theta(\eta)+\Theta(-\eta)],
\end{aligned}
$$

where $\Theta$ is the step function, and $\eta^{\mu \nu}$ is the Minkowski metric. The integration of the field equations

$$
G_{M}^{N}=\frac{4 \pi}{L_{p}^{(3-d)}} T_{M}^{N}
$$

across the boundary $\partial \Omega$ yields,

$$
\Delta \mathfrak{K}_{\mu}^{\nu}-\delta_{\mu}^{\nu} \Delta \mathfrak{K}=\frac{4 \pi}{L_{p}^{(3-d)}} \sigma \delta_{\mu}^{\nu},
$$

where

$$
\Delta \mathfrak{K}_{\nu}^{\mu} \equiv \lim _{\epsilon \rightarrow 0} \mathfrak{K}_{\nu}^{\mu^{(-)}}-\mathfrak{K}_{\nu}^{\mu^{(+)}},
$$

is the jump in the second fundamental form of the brane in going from $-\epsilon$ to $+\epsilon$ side. For the case at hand $\Delta \mathfrak{K}_{\mu}^{\nu}$ $=2 \mathfrak{K}_{\mu}^{\nu^{(-)}}$.

In order to analyze the dynamics of the system, the brane is allowed to move radially. Let the position of the brane be described by $x^{\mu}\left(\tau, \phi_{i}\right) \equiv\left(t(\tau), a(\tau), \phi_{i}\right)$, with $\tau$ the proper time (as measured by co-moving observers on the brane) that parametrizes the motion, and the velocity of a piece of stress energy at the brane satisfying $u_{M} u^{M}=-1$. Note that, with these assumptions the brane will have an "effective scale factor" $\mathcal{A}^{2}(t)=a^{2}(t) A^{2}(t)$.

The unit normal to the brane reads

$$
\hat{n}^{M}=\left[\frac{a^{\prime} A}{\left(1-k a^{2}\right)^{1 / 2}}, 0, \ldots, 0, \frac{1}{A}\left[1-k a^{2}+\left(a^{\prime} A\right)^{2}\right]^{1 / 2}\right] .
$$

Using the identity $\partial_{\eta}=\hat{n}^{M} \partial_{M}$, plus the standard relation

$$
\mathfrak{K}_{\tau}^{\tau}=\frac{\partial x^{M}}{\partial x^{\tau}} \frac{\partial x^{\tau}}{\partial x^{N}} \mathfrak{K}_{N}^{M},
$$

the non-trivial components of the extrinsic curvature are given by

$$
\mathfrak{K}_{\phi_{i}}^{\phi_{i}}=\frac{\dot{A} a^{\prime}}{\left(1-k a^{2}\right)^{1 / 2}}+\frac{1}{a A}\left[1-k a^{2}+\left(a^{\prime} A\right)^{2}\right]^{1 / 2},
$$

and

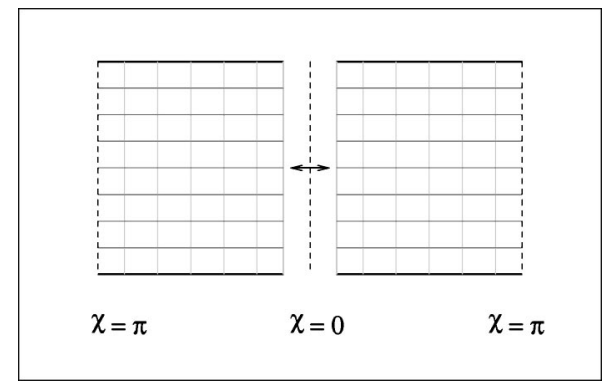

FIG. 1. Penrose diagram of dS spacetime with a spherical domain wall. Double arrow stands for identification. Horizontal (vertical) inner lines are $t-(\chi-)$ constant surfaces. The $t= \pm \infty$ surfaces correspond to the top and bottom horizontal lines, respectively. Vertical dashed lines represent the coordinate singularities $\chi=0$ and $\chi=\pi$, typical of polar coordinates. We have used the transformation $r=\sin (\chi)$.

$$
\begin{aligned}
\mathfrak{K}_{\tau}^{\tau}= & \frac{a^{\prime \prime} A}{\left[1-k a^{2}+\left(a^{\prime} A\right)^{2}\right]^{1 / 2}}+\frac{2 a^{\prime} \dot{A}}{\left[1-k a^{2}\right]^{1 / 2}} \\
& +\frac{2 a^{\prime 2} k a A}{\left[1-k a^{2}+\left(a^{\prime} A\right)^{2}\right]^{1 / 2}\left[1-k a^{2}\right]},
\end{aligned}
$$

where $a^{\prime} \equiv d a / d \tau$, and $\dot{A} \equiv d A / d t$. The proper time is related to the coordinate time by

$$
\frac{d \tau}{d t}= \pm \sqrt{1-\frac{(A \dot{a})^{2}}{1-k a^{2}}}
$$

Thus, one can always eliminate one in favor of the other by using $a^{\prime}=\dot{a} d t / d \tau$. With this constraint Eqs. (5) and (6) become two sets of differential equations relating unknown functions of $t: A, a$. In the following subsections we find out particular solutions of this system.

\section{B. dS}

Let us consider two patches of dS spacetimes undergoing expansion. This implies setting $A(t)=l \cosh (t / l)$ and $k=1$ in Eq. (2), where $l^{2}=d(d-1) /|\Lambda|$ is the dS radius. Figure 1 shows the Penrose diagram of the model considered in this subsection.

As usual, there is a redundancy between the field equations and the covariant conservation of stress-energy. A straightforward calculation shows that the equation of motion of the brane reads

$\frac{4 \pi}{L_{p}^{(3-d)}(d-1)} \sigma=\frac{ \pm \dot{a} \sinh (t / l)+[a l \cosh (t / l)]^{-1}\left(1-a^{2}\right)}{\left\{1-a^{2}-[l \cosh (t / l) \dot{a}]^{2}\right\}^{1 / 2}}$

We study first the case $\sigma=0$. Integration of Eq. (13) yields

$$
a(t)=\sqrt{\mathcal{C} \tanh ^{2}(t / l)+1},
$$

and 


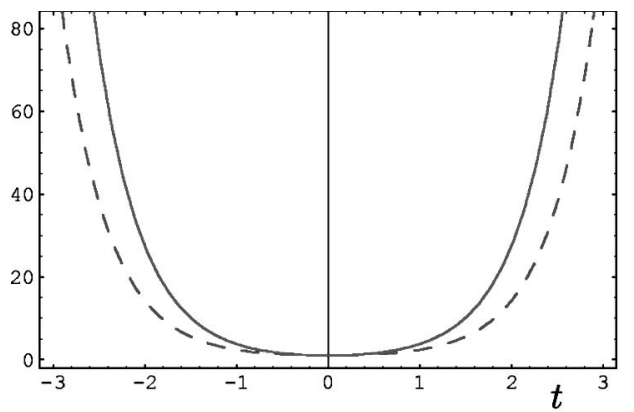

(i)

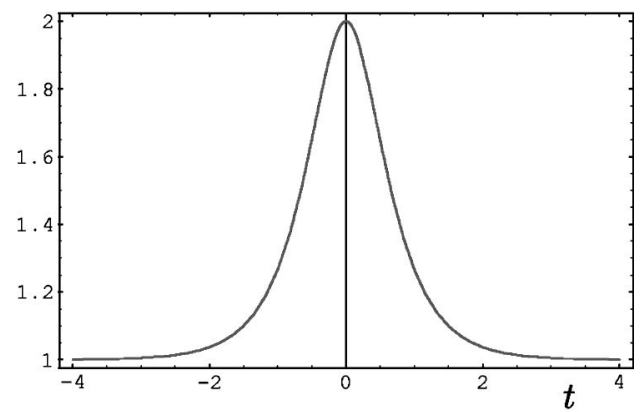

(ii)

$$
a(t)=\sqrt{\mathcal{C} \operatorname{coth}^{2}(t / l)+1},
$$

for positive and negative sign in Eq. (13), respectively. $\mathcal{C}$ is an integration constant which we will set to 1 in what follows. Note that the proper time is always real for both solutions.

Let us consider first Eq. (14). A plot of the effective scale factor for this case, Fig. 2(i), shows that it corresponds to an eternal non-singular universe that undergoes a phase of accelerated contraction up to a minimum volume at $t=0$, and then expands again. Note that, for all $t$ the expansion of the bulk dominates the cosmological evolution of the brane.

If we define the Hubble parameters $H_{\text {brane }}=2[\dot{a} / a$ $+\dot{A} / A]$ and $H_{\text {bulk }}=2 \dot{A} / A$, we can compare the rates of expansion by plotting $\Upsilon=H_{\text {brane }} / H_{\text {bulk }}$. This is shown in Fig. 2(ii). For $t \rightarrow-\infty$, both the brane and the bulk are infinitely large and start contracting at equal rates. Later, the contraction of the brane is faster than the one of the bulk, until the minimum is reached. The subsequent evolution is the mirror image of the evolution from $-\infty$ to 0 . The weighted rate of evolution (expansion or contraction) is larger on the brane for all $t$. For $|t| \ll 1$ the rate of expansion on the brane $H_{\text {brane }}$ is decoupled from that on the bulk with a behavior given by $\Upsilon \sim 2-2 t^{2}+10 t^{4} / 3+\mathcal{O}\left(t^{5}\right)$. For large values of $t, \mathcal{Y}$ $=2 \cosh ^{2}(t) \operatorname{sech}(2 t)$.

Now, we turn to the analysis of the second solution given by Eq. (15). As in the previous case, both the bulk and the brane have infinite volume at $t \rightarrow-\infty$, see Fig. 3(i). Due to the fact that $a(t)$ is finite for $\lim _{t \rightarrow \pm \infty}$, asymptotically the expansion of the bulk will dominate again the evolution of the spacetime. However, as $t$ approaches zero, $\operatorname{coth}(t)$ diverges, so that $a(t)$ drives the evolution of the brane. The behavior of $Y$ is plotted in Fig. 3(ii). This universe is com- posed of two disconnected branches, symmetric with respect to $t=0$. The branch on the left starts with an infinite volume, contracts up to a minimum, and then re-expands back to infinite volume. It should be stressed that near $t=0$, a finite interval of the coordinate time $t$ is associated to an infinite interval of proper time.

Notice that both solutions exhibit a bounce. They can be understood also as a special class of wormhole, a Tolman wormhole [28], which entails a violation of the strong energy condition on the brane.

In order to obtain a solution for any $\sigma>0$ we must solve Eq. (13) numerically. For simplicity we set $l=1$. After a brief calculation, Eq. (13) (keeping only the + sign) can be cast as

$$
A(a, t) \dot{a}^{2}+B(a, t) \dot{a}+C(a, t)=0,
$$

where

$$
\begin{aligned}
& A(a, t)=a^{2} \cosh ^{2}(t)\left[\overline{\sigma^{2}} \cosh ^{2}(t)+\sinh ^{2}(t)\right], \\
& B(a, t)=\left(1-a^{2}\right) a \sinh (2 t), \\
& C(a, t)=\left(1-a^{2}\right)^{2}-\overline{\sigma^{2}} \cosh ^{2}(t) a^{2}\left(1-a^{2}\right),
\end{aligned}
$$

and the factor $4 \pi /\left[L_{p}^{(3-d)}(d-1)\right]$ has been absorbed in $\bar{\sigma}$. From Eq. (16) it is straightforward to write

$$
\dot{a}=\frac{-B(a, t) \pm \sqrt{B^{2}(a, t)-4 A(a, t) C(a, t)}}{2 A(a, t)}, \quad a\left(t_{0}\right)=a_{0} .
$$

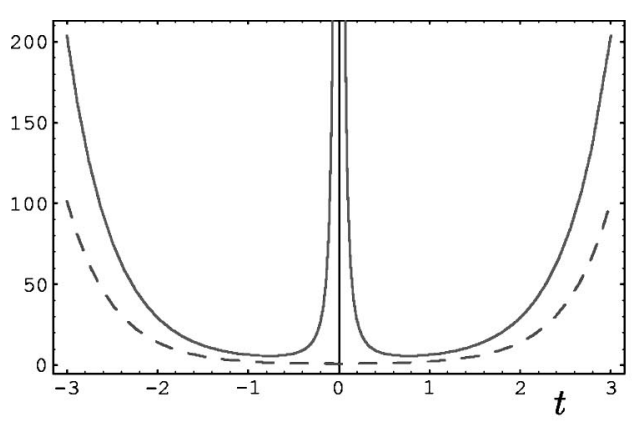

(i)

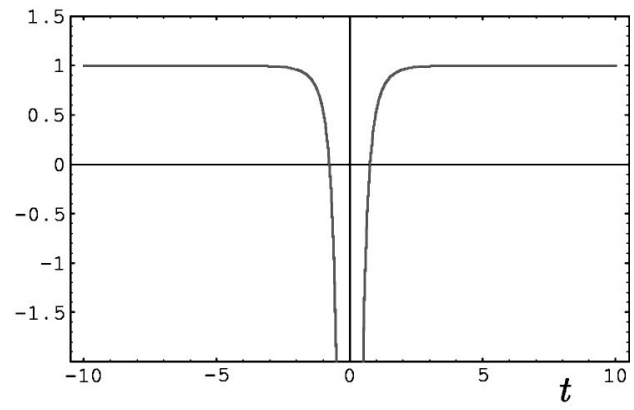

(ii)
FIG. 3. (i) Brane (solid-line) and bulk (dashed-line) scale factors. (ii) Ratio between the brane and bulk expansions, $Y(t)$. We chose $l=1$ and $\sigma=0$, as in the previous case. 


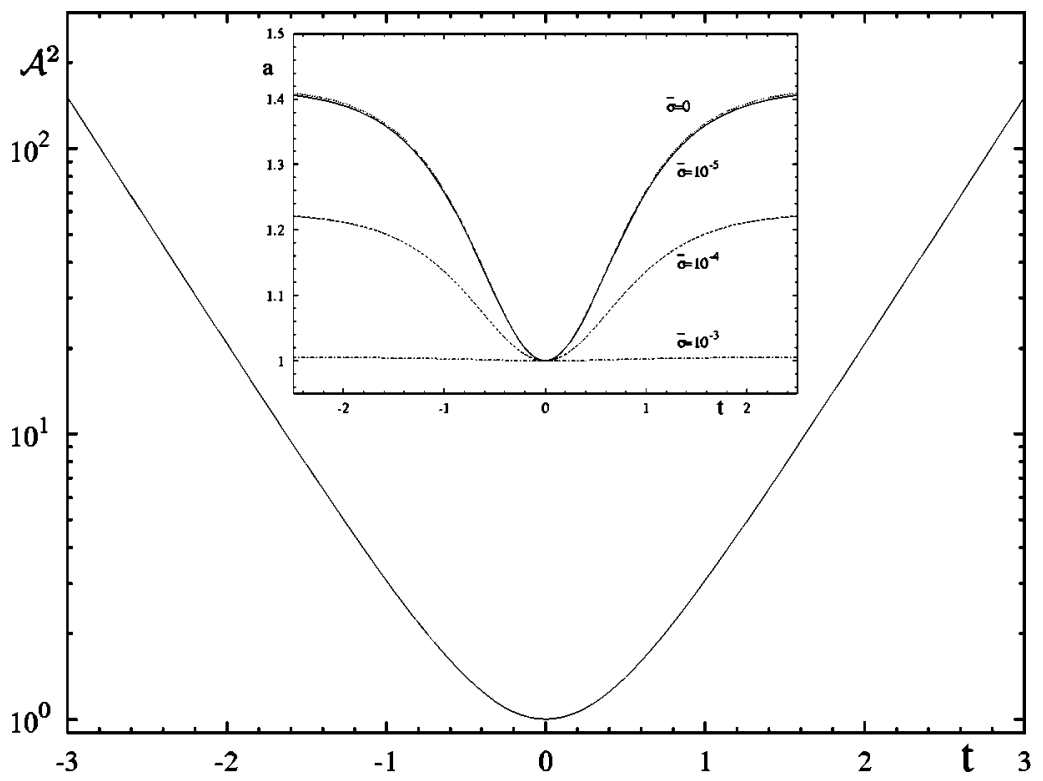

FIG. 4. The "effective scale factor" $\mathcal{A}^{2}(t)$ is plotted for $\bar{\sigma}=10^{-4}$. The embedded figure displays the dependence of $a(t)$ with $\bar{\sigma}$ (the imaginary part is zero).

It must be noted here that Eq. (20) represents two different initial value problems due to the \pm sign in front of the square root. From the discussion above, one would wish that $a(0)=1$, when $\sigma \rightarrow 0$. However, this does not lead to a well defined problem. In order to overcome this step, we take $t_{0}$ close to 0 (positive or negative). This yields $a_{0}$ near 1 as desired. For the sake of numerical accuracy [29] the right hand side of the above equation must be written in a different-but equivalent-way. Assuming that $a$ is a complex-valued function, we get the following initial value problems:

$$
\dot{a}=\frac{-B(a, t)-q \sqrt{B^{2}(a, t)-4 A(a, t) C(a, t)}}{2 A(a, t)}, \quad a\left(t_{0}\right)=a_{0},
$$

and

$$
\dot{a}=\frac{-2 C(a, t)}{B(a, t)+q \sqrt{B^{2}(a, t)-4 A(a, t) C(a, t)}}, \quad a\left(t_{0}\right)=a_{0},
$$

where $q=1$ if

$$
\operatorname{Re}\left\{B^{*}(a, t) \sqrt{B^{2}(a, t)-4 A(a, t) C(a, t)}\right\} \geqslant 0,
$$

or $q=-1$ otherwise.

Solutions of Eq. (22) for different values of $\bar{\sigma}$ are shown in Fig. 4. They were obtained using the well-known fourthorder Runge-Kutta method [30]. In order to check the results obtained numerically, we consider the expansion $a(t)=a_{0}$ $+a_{1} t+\mathcal{O}\left(t^{2}\right)$ around $t=0$. By replacing it in Eq. (13) we get

$$
\begin{aligned}
& a_{0}^{2}=\frac{1}{2 \overline{\sigma^{2}}}\left(\sqrt{1+4 \overline{\sigma^{2}}}-1\right), \\
& a_{1}^{2}=1-2 a_{0}^{2}+a_{0}^{4} .
\end{aligned}
$$

For instance, it is straightforward to see that if $\bar{\sigma}=10^{-3}$, the slope goes to zero.

As in the case $\sigma=0$, the model describes an eternal nonsingular universe. By progressively increasing the tension of the brane we obtain slower "cosmological" developments. The effective cosmological constant on the brane is given by

$$
\lambda_{\mathrm{eff}}=\frac{2}{3} \frac{d-1}{d} \Lambda+\frac{1}{8}(d-2)(d-1) \overline{\sigma^{2}} .
$$

It is interesting to remark that by modifying the vacuum energy of the bulk, one can obtain a similar evolution for $a$. In other words, by increasing the dS radius $l, a(t)$ becomes smoother as depicted in Fig. 5. Thus, one can say that $\Lambda$ and $\sigma$ have opposite effects.

At this stage, it is worth noting that the tidal acceleration in the $\hat{n}^{M}$ direction as measured by observers on the brane with velocity $u^{N}$ is given by $-\hat{n}_{M} R^{M}{ }_{N O P} u^{N} \hat{n}^{O} u^{P}$, where $R^{M}{ }_{N O P}$ is the Riemann tensor. Recalling that we are dealing with a conformally flat bulk,

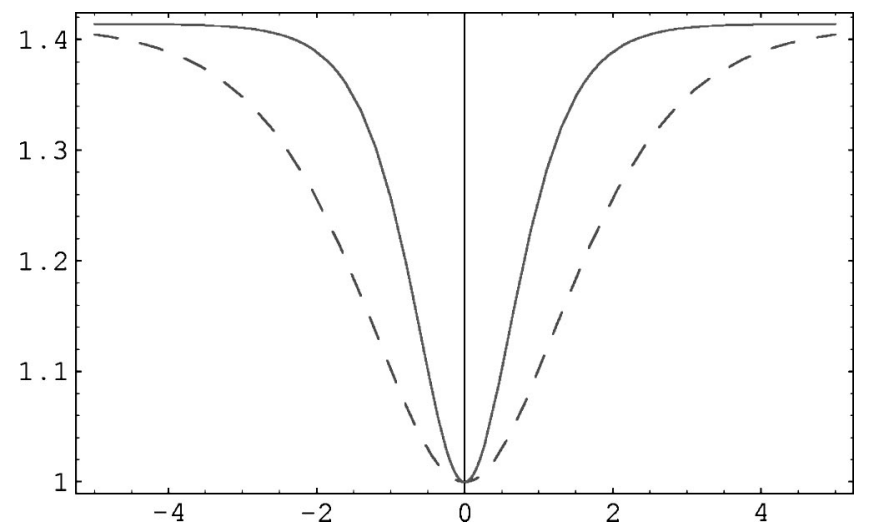

FIG. 5. Comparison of $a(t)$ for different dS radius. The solid line stands for $l=1$, whereas the dashed line is for $l=2$. $\sigma$ was set to zero. 


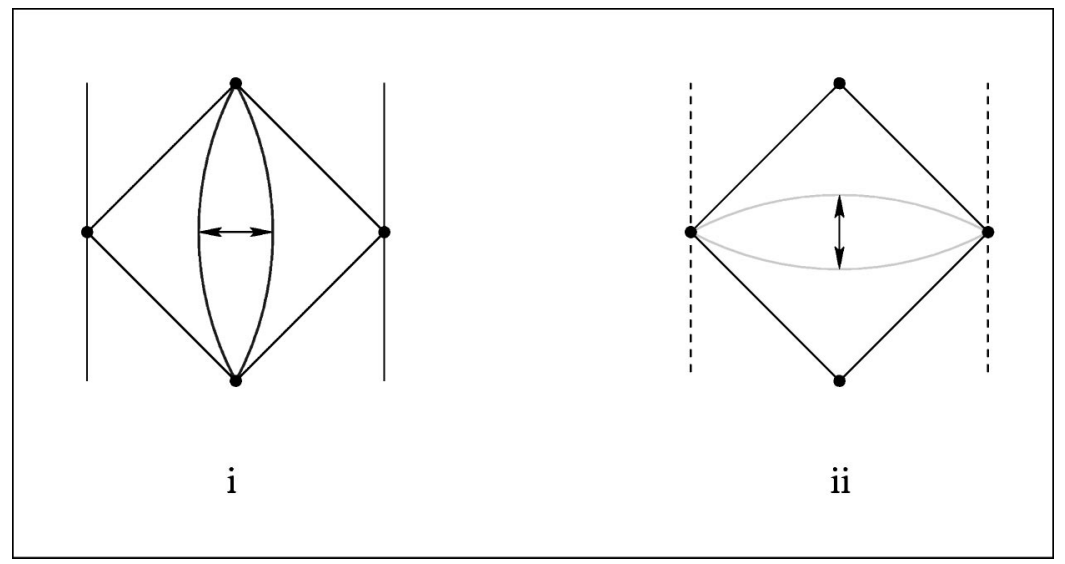

FIG. 6. (i) Penrose diagram of AdS space with a dS-RS-type domain wall. The arrows denote identifications. The vertical solid lines represent timelike infinity. (ii) AdS space with a dS domain wall and pre-surgery regions inside the lightcone. The vertical dashed-lines denote spacelike infinity.

tidal acceleration in off-brane direction $\propto \Lambda$.

Consequently, a positive $\Lambda$ furnishes an acceleration away from the brane, giving rise to a non-localized gravity model [31]. This situation may change when considering models with non-local bulk effects (through a nonzero Weyl tensor in the bulk [16]), or with the incorporation of moduli fields [32] (see the Appendix). Note that one can compactify the spacetime by adding a second brane. In order to do this, one must redefine the jump in the second fundamental form (in going from $-\epsilon$ to $\epsilon$ ) between the two new disjoint boundaries $\partial \Omega_{i} \equiv\left\{r_{i}=b<a\right\}$ as

$$
\Delta \mathfrak{K}_{\nu}^{\mu} \equiv \lim _{\epsilon \rightarrow 0} \mathfrak{K}_{\nu}^{\mu^{(+)}}-\mathfrak{K}_{\nu}^{\mu^{(-)}},
$$

and then repeat the entire computation after the necessary changes have been made [33].

Minkowski or dS compactifications seem to be ruled out by some results from low energy string theory [34]. However, all the "no go" arguments rely on the low energy limit of type II string theory (or M theory), where negative tension objects do not exist. The full string theory could provide objects such as orientifold planes [35], and compactification to RS setups after including string-loop corrections to the gravity action. In addition, massive type II A supergravity could be an alternative arena for realizing dS compactifications or RS models on manifolds with boundary. Steps in this direction were presented elsewhere [36]. A different approach was discussed in [37]. All in all, if the bulk is conformally flat, then it is the sign of the bulk cosmological constant that determines whether there is gravity trapping or not. Henceforth, we study AdS bulks.

\section{AdS}

By setting $A(t)=\sin (t), k=-1, l=1$ in Eq. (2), it is obtained

$$
d s^{2}=-d t^{2}+\sin ^{2}(t)\left[r^{2} d \Omega_{(d-1)}^{2}+\left(1+r^{2}\right)^{-1} d r^{2}\right] .
$$

This is the metric associated to the universal covering space of AdS. The above metric only covers a part of the universal covering space, even when all the intervals $t \in[n \pi,(n$
$+1) \pi$ ] (for any integer $n$ ) are included. Let us consider only one of the possible patches for the coordinate $t$ ranging between 0 and $\pi$. At $t=\pi$ there is a Cauchy horizon connecting to a new spacetime that is physically unreachable. This horizon is the light-cone emanating from the center of symmetry of the solution. Before proceeding further, it is worthwhile to point out that by means of the change of variables $t=i \rho, r=\sinh (i \zeta)$, one obtains the analytic continuation of Eq. (28) that reads

$$
d s^{2}=d \rho^{2}+\sinh ^{2}(\rho)\left[d \zeta^{2}+\sin ^{2}(\zeta) d \Omega_{(d-1)}^{2}\right] .
$$

In addition, the following coordinate transformations:

$$
\begin{aligned}
\frac{d \alpha}{d \phi_{(d-1)}} & =\tanh (\rho) \sin (\zeta) \sin \left(\phi_{i}\right) \sin \left(\phi_{j}\right) \ldots \sin \left(\phi_{(d-2)}\right), \\
\frac{d y}{d \rho} & =\left(1+y^{2}\right)^{1 / 2},
\end{aligned}
$$

entail a diffeomorphism between the Euclidean AdS-metric given in Eq. (29) and

$$
d s^{2}=\left(1+y^{2}\right) d \alpha^{2}+\left(1+y^{2}\right)^{-1} d y^{2}+y^{2} d \Omega_{(d-1)}^{2} .
$$

This metric covers the whole spacetime. Finally, the analytic continuation of Eq. (31) into real time $(\alpha=i T)$ leads to the AdS region outside the light-cone (Rindler horizon). It is important to stress that the identification between the two Lorentzian metrics involves an analytic continuation. Notice that although the $(d+1)$-dimensional part of the gravity action is boundary independent, the last two terms in Eq. (1) do indeed depend on the choice of the boundary setup. Unlike standard RS-type scenarios [38], we proceed here by excising two spacetime regions internal to the Rindler horizon and gluing the two copies along the $(d-1)$-dimensional spheres. The key difference between both scenarios is shown in Fig. 6 (more on this below).

With this in mind, the equation of motion of a clean brane sweeping AdS is given by

$$
\frac{4 \pi \sigma}{L_{p}^{(3-d)}(d-1)}=\frac{ \pm \dot{a} \cos (t)+[a \sin (t)]^{-1}\left(1+a^{2}\right)}{\left\{1+a^{2}-[\sin (t) \dot{a}]^{2}\right\}^{1 / 2}} .
$$




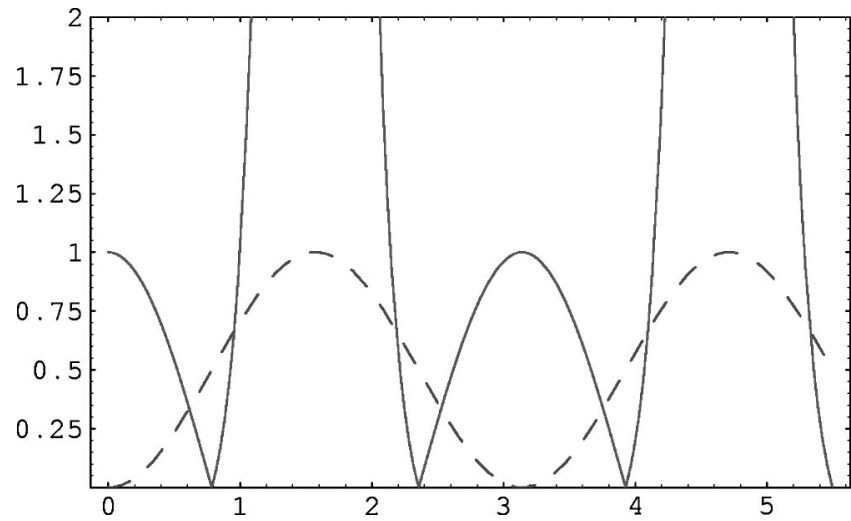

FIG. 7. Brane (solid-line) and bulk (dashed-line) scale factors as a function of $t$ with $\mathcal{C}=1$.

For $\sigma=0$, straightforward integration yields

$$
a(t)= \begin{cases}\sqrt{\mathcal{C} \cot ^{2}(t)-1} & \text { for } t \in(0, \pi / 4] \\ \sqrt{\mathcal{C} \tan ^{2}(t)-1} & \text { for } t \in(\pi / 4, \pi / 2]\end{cases}
$$

To obtain this solution we have exchanged the \pm sign in the equation of motion at $t=\pi / 4$. In Fig. 7 we show the evolution of the bulk and brane scale factors for $t \in[0,7 / 4 \pi]$. Note however, that Eq. (33) does not describe a physical solution because the proper time is not real. By checking the Jacobian in Eq. (12) one can immediately realize that the intervals where the proper time is real are shifted in $\pi / 2$ with respect to the corresponding solutions of Eq. (33). To understand what is going on, let us re-write the effective cosmological constant on the brane,

$$
\lambda_{\mathrm{eff}}=(d-1)\left[\frac{1}{8}(d-2) \overline{\sigma^{2}}-\frac{2}{3} \frac{(d-1)}{l^{2}}\right] .
$$

It is easily seen that in order to obtain $\lambda_{\text {eff }}>0$ [a condition that arises from imposing that the sections $t=$ const and $a$ $=$ const are $(d-1)$ spheres], we must impose

$$
\overline{\sigma^{2}}>\frac{16}{3} \frac{(d-1)}{(d-2)} \frac{1}{l^{2}} \text {. }
$$

Thus, the existence of a well behaved solution of a spherical domain wall sweeping the internal region to the Rindler horizon has an explicit dependence on the dimension of the spacetime through the brane tension.

In order to find the solution for $\bar{\sigma}>0$ we follow the procedure sketched before for the case of a dS bulk. In Fig. 8 we show the solution of Eq. (32) (considering again only the plus sign) for $\bar{\sigma}=2$. It has been obtained by setting $l=1$, and with the initial condition $a(1)=1$. For comparison in Fig. 9 we display the corresponding analytical solution of Eq. (33), for $t \in(\pi / 4, \pi / 2]$. One can check by inspection that $a(t)$ becomes smoother with increasing $\bar{\sigma}$. This implies that the denominator on the r.h.s. of Eq. (32) is real, rendering a well defined proper time. Putting all this together, a non-vanishing brane tension leads to the suitable shifting on $a(t)$.

\section{NON-PERTURBATIVE STRING COSMOLOGY VIA AdS/CFT}

A seemingly different, but in fact closely related subject that we will discuss in this section is the AdS/CFT correspondence [39]. This map provides a "holographic" projection of string theory (or M theory) in AdS space, to a conformal field theory (CFT) living on its boundary [40]. Actually, more general spaces with certain smooth restrictions would typically lead to nonconformal field theories on their boundary. For asymptotically AdS spaces the bulk excitations do indeed have a correspondent state/operator in the boundary [41]. The duality between the "strongly coupled gauge theory/weakly coupled gravity" is the face-off of the well known computation of black hole quantities via a field theory [42] that naturally yields a non-perturbative stringy background. In the standard non-compact AdS/CFT setup, gravity is decoupled from the dual boundary theory. However, any RS-like model should properly be viewed as a cou-

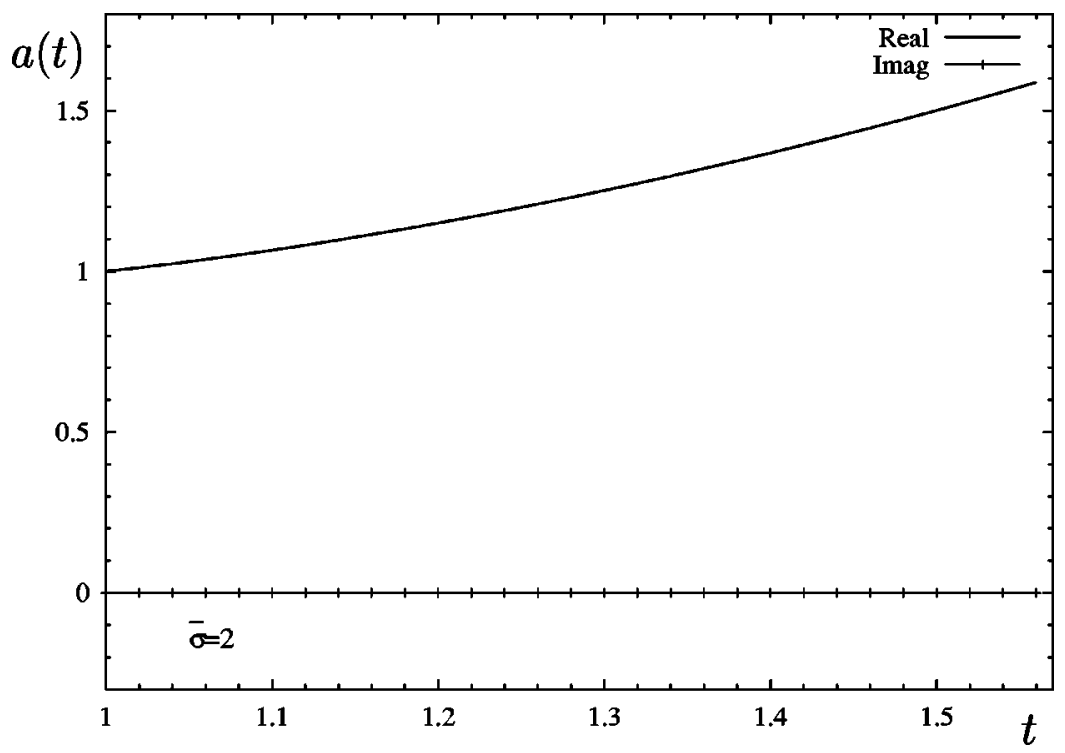

FIG. 8. Real and imaginary parts of $a(t)$ for $\bar{\sigma}=2$. 


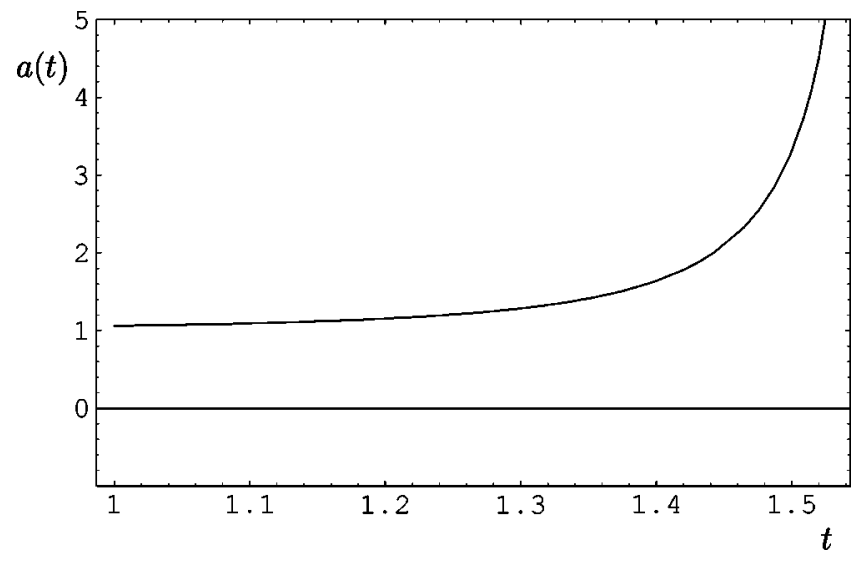

FIG. 9. Analytical solution for $\bar{\sigma}=0$ from Eq. (33) for $t$ $\in(\pi / 4, \pi / 2]$. We have considered $\mathcal{C}=5 \times 10^{-2}$, so as to approximately reproduce the initial condition of the numerical solution depicted in Fig. 8.

pling of gravity to whatever strongly coupled conformal theory the AdS geometry is dual to [43]. The holographic description has been recently invoked to discuss phenomenological and gravitational aspects of RS-models [44]. Here, we try to take advantage of this duality to describe cosmological setups.

There exists a "new lore" that convinces us that if our universe is five-dimensional, it should have evolved to the present situation from $\mathrm{M}$ theory [45]. If this is the case, it is reasonable to describe the evolution of the $\mathrm{AdS}_{5}$ sector (up to stabilization at some scale) by

$$
d s^{2}=-d t^{2}+\sin ^{2}(t)\left[\sinh ^{2}(\zeta) d \Omega_{(d-1)}^{2}+d \zeta^{2}\right] .
$$

In other words, the metric in Eq. (36) with $S O(d, 1)$ isometries [which is in fact a subgroup of the full $S O(d, 2)$ symmetry group of AdS] is expected to characterize the dynamics of the system that has "stationary" phases governed by

$$
d s^{2}=-\left(1+y^{2}\right) d T^{2}+\left(1+y^{2}\right)^{-1} d y^{2}+y^{2} d \Omega_{(d-1)}^{2} .
$$

Recall that we set $l=1$, and the discussion refers to $d=4$. This overall picture is actually related to the notion of black holes threaded with collapsing matter, stabilized as static objects. Particularly, in such a limit the dual non-perturbative description for maximally extended Schwarzschild-AdS spacetimes has been recently put forward [46]. Applying Maldacena's conjecture in the whole dynamical scenario, however, is not straightforward. Basically, because the well known correspondence between correlation functions in a field theory and string theory backgrounds with AdS subspace [41] does not have a clear counterpart. We are not going to present here a prescription for such a generalization, which is beyond the scope of the present article. Instead, we content ourselves with assuming the existence of a subset of operators/states satisfying certain discrete symmetries, and sketch a CFT dual of the dynamical gravitational system discussed in the previous section.

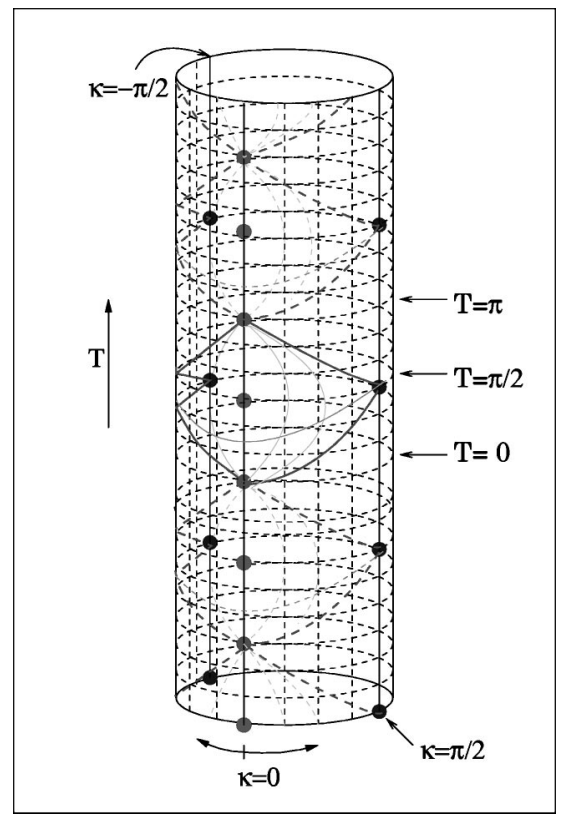

FIG. 10. A chain of diamond-shaped regions of the universal covering of the AdS. Here, $\kappa=2 \arctan [\exp (\rho)]-\pi / 2$.

In order to do so, we should first draw the reader's attention to some generic features of the metrics in Eqs. (36) and (37). The $(p+2)$-dimensional AdS space can be obtained by taking a hyperboloid (for instance see [47])

$$
-x_{0}^{2}-x_{p+2}^{2}+\sum_{j=1}^{p+1} x_{j}^{2}=-1
$$

embedded in $(p+3)$-dimensional space with a metric

$$
d s^{2}=-d x_{0}^{2}-d x_{p+2}+\sum_{j=1}^{p+1} d x_{j}^{2} .
$$

By construction, the spacetime contains "anomalies" in the form of closed time-like curves, corresponding to the $\mathrm{S}^{1} \mathrm{sec}$ tor of the hyperboloid. However, by unwrapping that circle one can eliminate the causal "anomalies," and obtain the so called "universal covering space of AdS." A convenient parametrization sets $x_{p+2}=\cos (t)$, so that a constant $t$ surface is a constant negative curvature hyperboloid of radius $\sin (t)$, with the metric given in Eq. (36). One can alternatively solve Eq. (38) by setting

$$
\begin{aligned}
& x_{0}=\cosh (y) \sin (T), \quad x_{p+2}=\cosh (y) \cos (T), \\
& x_{j}=\sinh (y) \Omega_{j},
\end{aligned}
$$

where $j=1, \ldots, p+1$, and $\Sigma_{j} \Omega_{j}^{2}=1$. By inspection of Fig. 10 , it is easily seen that the universal AdS space is conformal to half of the Einstein static universe. While the coordinate system $\left(t, \zeta, \phi_{i}\right)$, with apparent singularities at $t=n \pi(n$ $\in Z$ ), covers only diamond-shaped regions, coordinates $\left(T, y, \phi_{i}\right)$ cover the whole space. The surface corresponding to $t=-\infty$ (solid line) emanates from $T=0$ bouncing at $T$ $=\pi / 2$ towards $T=\pi$. After reflection, the solid line repre- 
sents a hypersurface at $t=\infty$. The vertical thin solid lines (with end points $T=0, T=\pi$ ) stand for hypersurfaces $y$ $=$ const, whereas the corresponding horizontal lines are $T$ $=$ const curves. Notice that every timelike geodesic emanating from any point in the space (to either past or future) reconverges to an image point, diverging again to refocus at a second image point, and so on [48]. Therefore, the sets of points which can be reached by future directed timelike lines starting at a point $p$, is the set of points lying beyond the future null cone of $p$, i.e., the infinite chain of diamond shaped regions similar to the one characterized by $\left(t, \zeta, \phi_{i}\right)$. Since the total space is non-singular, it should be expected that the other regions that are outside the diamond-shaped domain can be included. All points in the Cauchy development of the surface $T=0$ can be reached by a unique geodesic normal to the surface, whereas points lying outside the Cauchy development cannot be reached by this kind of geodesics.

The infinite sequence of diamond-shaped regions can also be understood as quotients with respect to the universal covering space [23]. If we consider a quotient of the form $H_{d} / \Gamma$ (with a discrete group of invariances $\Gamma$ ) such that we get a finite volume region out of $H_{d}$, we could interpret this as a cosmological model with a big bang and a big crunch [49]. This cosmological model is a quotient space constructed by identification of points in FRW space under the action of a discrete subgroup $\Gamma$ of the manifold's isometry group $G$. Intuitively, we divide the space in different regions such that for any observer placed at $x_{0}$, the so-called Dirichlet domain is defined as the set of all points closer to $x_{0}$ than to $\tilde{\gamma} x_{0}$, where $\tilde{\gamma}$ is an element of the group $\Gamma$. Notice that the action of $\tilde{\gamma}$ 's shifts the outskirts of each point in a given diamondshaped region to another diamond-shaped domain. With this in mind, one can describe the universe we live in by a Dirichlet domain. Then, we can see the importance of the Cauchy surface mentioned above at $T=0$ in Eq. (36), since after imposing initial data on this surface we can cover the complete AdS space.

A particular example of this sort of phenomenon occurs in the extended RS-universe discussed in Sec. II. The envisaged surgery, shown in Fig. 11, starts with two asymptotic AdS regions, each one with two outer and inner horizons, that are then repeated an infinite number of times in the maximal analytic extension. The brane expansion starts out in the downmost AdS region, falling through the future outer horizon to the next incarnation of the universe. This process occurs in a finite time $t$. Since we have an AdS space and a quotient on it, one can naturally ask what the effect of this quotient is on the CFT. This question is of great interest since the resulting CFT would be a non-perturbative description of string cosmology. The idea is to construct a CFT with a Hilbert space invariant under $\Gamma$. These states would in principle describe linearized gravity modes in the cosmological background [50]. The main problem with this construction is that finding such a CFT would involve taking a quotient on the space on which the CFT lives. The interesting point discovered in [23] is that one can take a quotient on the operator and state spaces leading to a subset invariant under $\Gamma$. Before

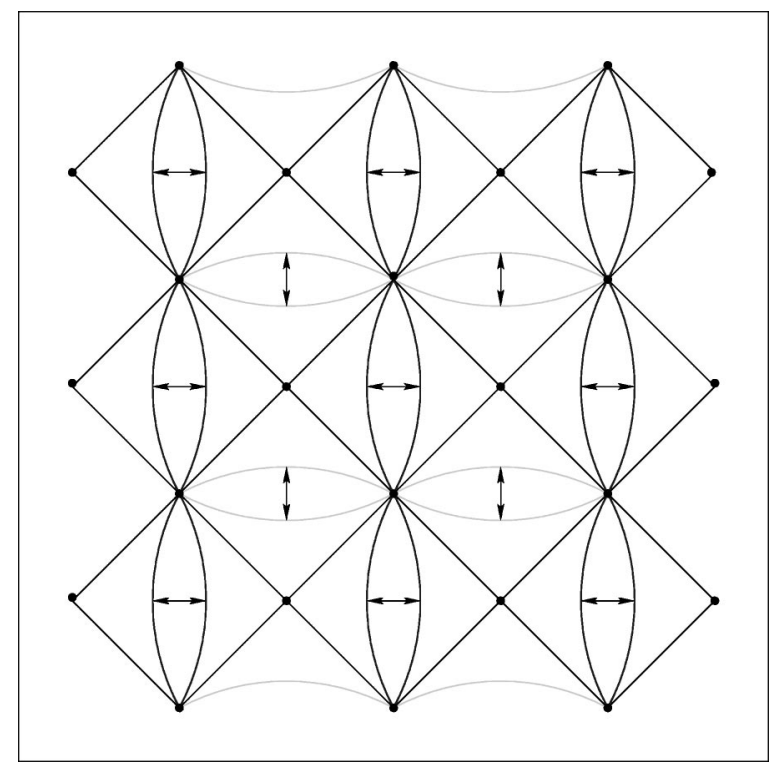

FIG. 11. Maximally extended Penrose diagram. The past and future RS horizons are replaced by the past and future light-cones obtained after analytical continuation.

proceeding further, it is instructive to recall that instead of a CFT living in a flat Minkowski space, we handle a gauge theory coupled to gravity on the brane.

When considering the near horizon geometry of D3 branes, $\mathrm{AdS}_{5} \times \mathrm{S}^{5}$, one deals with a four dimensional $\mathcal{N}=4$ supersymmetric Yang-Mills theory as its holographic dual. The symmetries on the brane side, $S L(2, Z)$ and $S O(6)$ are present on the gravity side as type IIB symmetries. An enormous number of tests on the above relation have been done. Many of them involve correlation functions. Others refer to the matching of the weight of chiral primaries on the CFT side with the masses of the KK modes on the compact part of the geometry. In the case of $\mathrm{M}$ theory, we deal with $\mathrm{AdS}_{4}$ $\times \mathrm{S}^{7}$ (and also with $\mathrm{AdS}_{7} \times \mathrm{S}^{4}$ ) and 3-dimensional supersymmetric CFT with 16 charges or the $(0,2)$ little string theory in each case (they correspond to M2 or M5 branes). In these cases the existence of a matching between chiral primaries and $\mathrm{KK}$ states was also checked in detail. The $\operatorname{AdS}_{6} \times \mathrm{S}^{4}$ (that appears when we consider the D4-D8 system in massive IIA theory [36]) leads to five-dimensional CFT. Again, the same matching and correspondence was achieved. Besides, there exist compactifications of $\mathrm{M}$ theory with an $\mathrm{AdS}_{5}$ sector (for instance $\mathrm{AdS}_{5} \times \mathrm{H}_{2} \times \mathrm{S}^{4}[51]$ ), where all the previous mentioned features work in pretty much the same way. The subtlety of these compactifications is that they lead to 4-dimensional CFT's with 4 or 8 supercharges, thus making closer contact with the supersymmetric SM-like theories.

In order to describe a method that can hopefully be applied to any of the products discussed above, let us restrict to the case of $\mathrm{AdS}_{3} \times \mathrm{S}^{3} \times \mathrm{T}^{4}$ (the background geometry that appears considering the D1-D5 system). The gravity system has a dual description in terms of a 2-dimensional supersymmetric CFT, with $\mathcal{N}=(4,4)$ supersymmetry. The isometries, given by $S L(2, R) \times S L(2, R)$, coincide with the symmetry generated on the 2-dimensional boundary by the Virasoro 
generators $L_{0, \pm}, \overline{L_{0, \pm}}$. A scalar field satisfying the KleinGordon equation in $\mathrm{AdS}_{3}$ will have eigenmodes in correspondence with the masses of the chiral primaries on the field theory side. These states, like all their descendants, are protected and in correspondence with the KK states on the $\mathrm{S}^{3} \times \mathrm{T}^{4}$ of the geometry. Once we have applied the quotient operation, the proposed way to obtain the correspondence is lifting the gravity mode to the uncompactified AdS space. This is carried out by considering a periodic function defined on each Dirichlet domain, covering the complete space. The correspondence is between the cosmological gravity mode, and the state given by the sum of individual states on each domain (on the CFT side). It is important to point out that this sum is not convergent. Therefore, methods dealing with rigged Hilbert spaces have been applied to define the sum (the CFT state) properly. In addition, as it has been pointed out in [23] the quotients required to obtain the cosmology break down all the supersymmetries. One can understand this considering that a spacetime where some of the supersymmetries remain unbroken must be stationary (or sort of "nullstationary"). However, our quotient spacetime has no Killing fields whatsoever. Note that such a Killing field would have to be an element of the AdS symmetry group that commutes with the group we used to take the quotient. However, there are no such elements. Another way of saying this is that, after the quotient, one will not be able to return (globally) to the static coordinate system [52]. It implies that there are no protected quantities in the cosmological scheme. Other ways of doing the quotient could in principle be found. This opens the possibility of different definitions of this theory in the boundary, which would be very interesting to explore further. Moreover, it is also important to address whether there could exist scenarios in which the above mentioned quotients describing the cosmological evolution do preserve some of the supersymmetries. We hope to return to these topics in future publications.

\section{ACKNOWLEDGMENTS}

We have benefitted from discussions with Juan Maldacena, Stephan Stieberger, and Mike Vaughn. We are grateful to Gia Dvali and Tetsuya Shiromizu for a critical reading of the manuscript and valuable comments. We would like to especially thank Gary Horowitz and Don Marolf for sharing with us their expertise on this subject and teaching us about their work. This article was partially supported by CONICET (Argentina), FAPERj (Brazil), Fundación Antorchas, IFLP, the Mathematics Department of the UNLP, the National Science Foundation (U.S.), and the U.S. Department of Energy (DOE) under cooperative research agreement no. DF-FC0294ER40818.

\section{APPENDIX: FLATLAND DYNAMICS}

In this appendix we will briefly discuss the main features of a novel scenario which contains fields in the bulk with Lagrangian $\mathcal{L}$. We start by considering a rather general action,

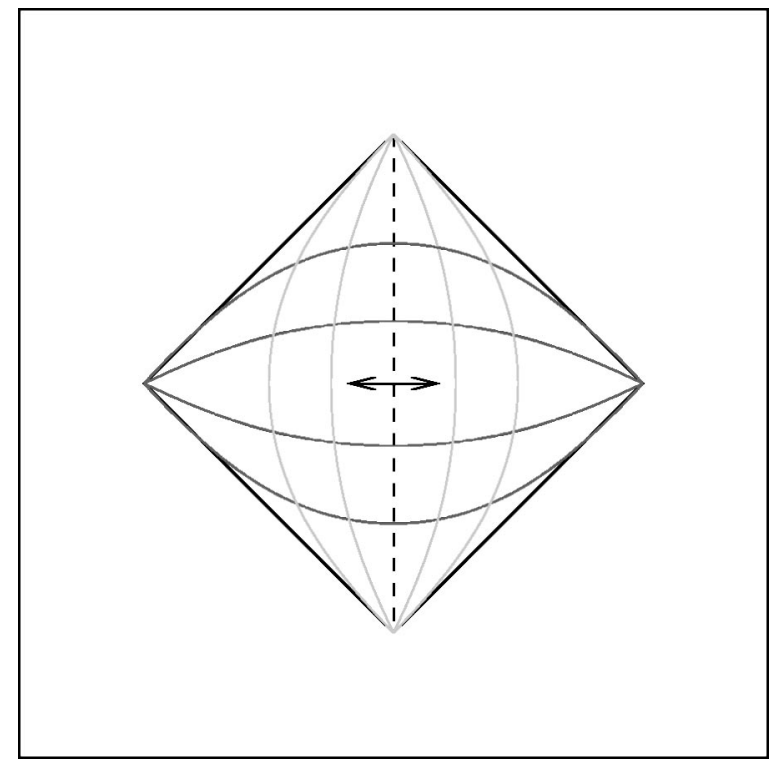

FIG. 12. Penrose diagram of Minkowski spacetime with a spherical domain wall. Double arrow stands for identification. Horizontal (vertical) inner lines are $t-(r-)$ constant surfaces. The $t$ $= \pm \infty$ surfaces correspond to the top and bottom horizontal lines, respectively. Vertical dashed line represents the coordinate singularities $r=0$, that occur with polar coordinates.

$$
\begin{aligned}
S= & \frac{L_{p}^{3-d}}{16 \pi} \int_{\mathcal{M}} d^{d+1} x \sqrt{g} R+\frac{L_{p}^{3-d}}{8 \pi} \int_{\partial \Omega} d^{d} x \sqrt{\gamma} \mathfrak{K} \\
& +\int_{\mathcal{M}} d^{d+1} x \sqrt{g} \mathcal{L}+\sigma \int_{\partial \Omega} d^{d} x \sqrt{\gamma} .
\end{aligned}
$$

The standard approach to solve the equations of motion would be to assume some specific type of fields descibed by $\mathcal{L}$, and from the physics of that source to derive an equation of state, which together with the field equations would determine the behavior of the $(d+1)$-dimensional scale factor. In deriving our model, however, the philosophy of solving Einstein's equation must be altered somewhat from the usual one: We concentrate on the geometrical properties of the spacetime, without specifying $\mathcal{L}$. We are interested here in $(d+1)$-dimensional Ricci-flat spacetime undergoing expansion. Thus, we set $k=0$ in Eq. (2) and we replace the metric in the field equations to obtain the curvature constraint

$$
R=2 d \frac{\ddot{A}}{A}+d(d-1) \frac{\dot{A}^{2}}{A^{2}} .
$$

Now, it is easily seen that

$$
A(t)=\left(\frac{\mathcal{K}_{0}}{2}\right)^{\alpha}\left[(1+d)\left(t-\mathcal{K}_{1}\right)\right]^{\alpha}
$$

describes a flat solution undergoing expansion, with equation of state (in terms of the bulk energy density $\rho$ and pressure p), 


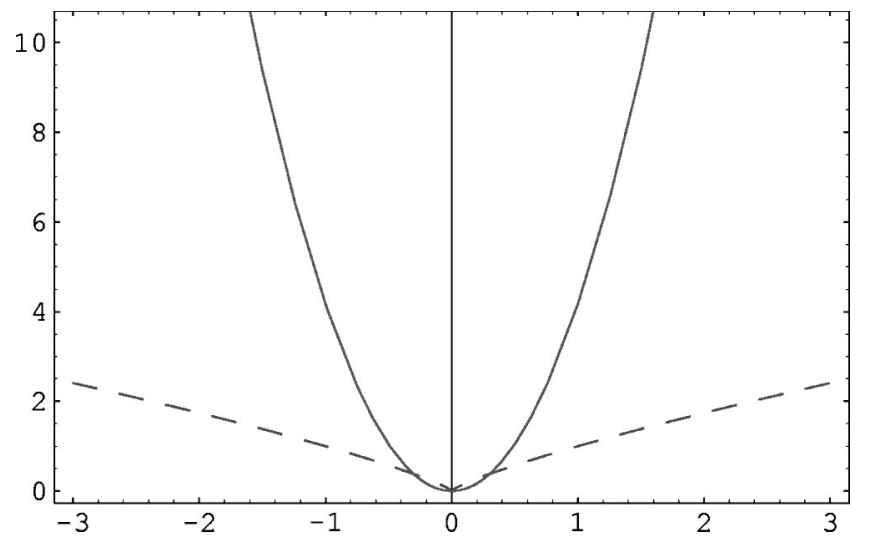

FIG. 13. Brane (solid-line) and bulk (dashed-line) scale factors, for $\sigma=0$.

$$
d \alpha(\alpha-1) t^{-2}=-\frac{4 \pi}{L_{p}^{3-d}}(\rho+d p),
$$

provided that $\alpha=2 /(d+1)$. For simplicity, we have set the integration constants $\mathcal{K}_{0}=\alpha, \mathcal{K}_{1}=0$. Note that the fields threading the spacetime violate the $(d+1)$ strong energy condition. After recalling that we are dealing with a clean brane and a conformally flat bulk, it is straightforward to obtain the equation of motion of a brane in this background,

$$
\frac{4 \pi}{L_{p}^{3-d}(d-1)} \sigma=\frac{ \pm \dot{a} \alpha t^{\alpha-1}+\left(a t^{\alpha}\right)^{-1}}{\left[1-\left(\dot{a} t^{\alpha}\right)^{2}\right]^{1 / 2}}
$$

Figure 12 shows the Penrose diagram for this brane-surgery. For the case $\sigma=0, d=4$ the solution reads,

$$
a(t)= \pm \frac{5}{\sqrt{6}} t^{3 / 5}
$$

where $a_{0}$ is an integration constant. In Fig. 13, we show the evolution of $A(t)$ and $\mathcal{A}(t)$ for $a_{0}=0$. The solution (brane and bulk scales factor) describes separating symmetric patches of contraction and expansion with a shrinkage down to zero at $t=0$. From Eq. (12) one can easily see that the solution does not represent a physical system because $(A \dot{a})^{2}>1$.

It is interesting to point out that in the most general action, the last world-volume term in Eq. (A1) would depend on the $d$-dimensional Ricci scalar. Such a term cannot be excluded by any symmetry reason and in fact is expected to be generated on the brane. Inclusion of this term often dramatically modifies the situation (e.g. can induce 4-dimensional gravity even if extra space is 5-dimensional Minkowskian [53]). Thus, it would be interesting to explore dynamical scenarios without fixing the shape of the brane.
[1] T. Kaluza, Sitzungsber. K. Preuss. Akad. Wiss. (Math. Phys.) K1, 966 (1921); O. Klein, Z. Phys. 37, 895 (1926) [Surv. High Energy Phys. 5, 241 (1926)].

[2] Variations of Kaluza-Klein theory that motivated the renaissance of higher dimensional models are discussed in, $\mathrm{K}$. Akama, Lect. Notes Phys. 176, 267 (1982); V.A. Rubakov and M.E. Shaposhnikov, Phys. Lett. 125B, 136 (1983); M. Pavsic, Class. Quantum Grav. 2, 869 (1985); Phys. Lett. 107A, 66 (1985); M. Visser, Phys. Lett. 159B, 22 (1985); G.W. Gibbons and D.L. Wiltshire, Nucl. Phys. B287, 717 (1987); I. Antoniadis, Phys. Lett. B 246, 377 (1990).

[3] G. Dvali and M. Shifman, Phys. Lett. B 396, 64 (1997); 407, 452(E) (1997); M. Gogberashvili, Mod. Phys. Lett. A 14, 2025 (1999); B. Bajc and G. Gabadadze, Phys. Lett. B 474, 282 (2000).

[4] L. Randall and R. Sundrum, Phys. Rev. Lett. 83, 4690 (1999).

[5] N. Arkani-Hamed, S. Dimopoulos, and G. Dvali, Phys. Lett. B 429, 263 (1998); I. Antoniadis, N. Arkani-Hamed, S. Dimopoulos, and G. Dvali, ibid. 436, 257 (1998); N. ArkaniHamed, S. Dimopoulos, and G. Dvali, Phys. Rev. D 59, 086004 (1999).

[6] Assuming that the higher dimensional theory at short distance is a string theory, one expects that the fundamental string scale $M_{s}$ and $M_{*}$ are not too different. A perturbative expectation is that $M_{s} \sim g_{s} M_{*}$, where $g_{s}^{2}$ is the gauge coupling at the string scale, of order $g_{s}^{2} / 4 \pi \sim 0.04$. S. Nussinov and R. Shrock, Phys. Rev. D 59, 105002 (1999).

[7] This scale emerges from supersymmetry breaking; see, for in- stance, L. Randall and R. Sundrum, Nucl. Phys. B557, 79 (1999), and references therein.

[8] Specifically, no deviations from Newtonian physics are expected at separations ranging down to $218 \mu \mathrm{m}$. C.D. Hoyle, U. Schmidt, B.R. Heckel, E.G. Adelberger, J.H. Gundlach, D.J. Kapner, and H.E. Swanson, Phys. Rev. Lett. 86, 1418 (2001).

[9] For an alternative approach to both large and small extra dimensions with asymmetric compactifications, see J. Lykken and S. Nandi, Phys. Lett. B 485, 224 (2000).

[10] See for instance, S. Cullen and M. Perelstein, Phys. Rev. Lett. 83, 268 (1999); N. Arkani-Hamed, S. Dimopoulos, G. Dvali, and N. Kaloper, J. High Energy Phys. 12, 010 (2000); V. Barger, T. Han, C. Kao, and R.J. Zhang, Phys. Lett. B 461, 34 (1999); G.C. McLaughlin and J.N. Ng, ibid. 470, 157 (1999); S. Cassisi, V. Castellani, S. Degl'Innocenti, G. Fiorentini, and B. Ricci, ibid. 481, 323 (2000).

[11] See for instance, G.F. Giudice, R. Rattazzi, and J.D. Wells, Nucl. Phys. B544, 3 (1999); T. Han, J.D. Lykken, and R. Zhang, Phys. Rev. D 59, 105006 (1999); J.L. Hewett, Phys. Rev. Lett. 82, 4765 (1999); E.A. Mirabelli, M. Perelstein, and M.E. Peskin, ibid. 82, 2236 (1999); T.G. Rizzo, hep-ph/9910255; S. Cullen, M. Perelstein, and M.E. Peskin, Phys. Rev. D 62, 055012 (2000); L. Anchordoqui, H. Goldberg, T. McCauley, T. Paul, S. Reucroft, and J. Swain, ibid. 63, 124009 (2001).

[12] L3 Collaboration, M. Acciarri et al., Phys. Lett. B 470, 281 (1999); H1 Collaboration, C. Adloff et al., ibid. 479, 358 (2000); DØ Collaboration, B. Abbott et al., Phys. Rev. Lett. 86, 1156 (2001). 
[13] Of particular phenomenological interest is the setup with the shape of a gravitational condenser in which two branes of opposite tension (which gravitationally repel each other) are stabilized by a slab of AdS. In this model the extra dimension is strongly curved, and the distance scales on the brane with negative tension are exponentially smaller than those on the positive tension brane. Such exponential suppression can then naturally explain why the observed physical scales are so much smaller than the Planck scale. L. Randall and R. Sundrum, Phys. Rev. Lett. 83, 4690 (1999).

[14] W.D. Goldberger and M.B. Wise, Phys. Rev. Lett. 83, 4922 (1999); J. Garriga and T. Tanaka, ibid. 84, 2778 (2000); N. Arkani-Hamed, S. Dimopoulos, G. Dvali, and N. Kaloper, ibid. 84, 586 (2000); J. Lykken and L. Randall, J. High Energy Phys. 06, 014 (2000); I.I. Kogan, S. Mouslopoulos, A. Papazoglou, G.G. Ross, and J. Santiago, Nucl. Phys. B584, 313 (2000); N. Arkani-Hamed, S. Dimopoulos, N. Kaloper, and R. Sundrum, Phys. Lett. B 480, 193 (2000); I.I. Kogan and G.G. Ross, ibid. 485, 255 (2000); R. Gregory, V.A. Rubakov, and S.M. Sibiryakov, Phys. Rev. Lett. 84, 5928 (2000); I.I. Kogan, S. Mouslopoulos, A. Papazoglou, and G.G. Ross, Nucl. Phys. B595, 249 (2001); I.I. Kogan, S. Mouslopoulos, and A. Papazoglou, Phys. Lett. B 501, 140 (2001); K.A. Meissner and M. Olechowski, Phys. Rev. Lett. 86, 3708 (2001); C. Csaki, M.L. Graesser, and G.D. Kribs, Phys. Rev. D 63, 065002 (2001); A. Karch and L. Randall, J. High Energy Phys. 05, 008 (2001); Phys. Rev. Lett. 87, 061601 (2001); J. High Energy Phys. 06, 003 (2001); see also N. Arkani-Hamed, A.G. Cohen, and H. Georgi, Phys. Rev. Lett. 86, 4757 (2001), for a discrete extra dimension framework, and G. Dvali, G. Gabadadze, M. Kolanovic, and F. Nitti, hep-th/0106058, for a model with low energy quantum gravity scale.

[15] V.A. Rubakov, hep-ph/0104152.

[16] T. Shiromizu, K. Maeda, and M. Sasaki, Phys. Rev. D 62, 024012 (2000).

[17] M. Cvetic, S. Griffies, and H.H. Soleng, Phys. Rev. D 48, 2613 (1993); N. Arkani-Hamed, S. Dimopoulos, N. Kaloper, and J. March-Russell, Nucl. Phys. B567, 189 (2000); P. Binetruy, C. Deffayet, and D. Langlois, ibid. B565, 269 (2000); N. Kaloper, Phys. Rev. D 60, 123506 (1999); C. Csaki, M. Graesser, C. Kolda, and J. Terning, Phys. Lett. B 462, 34 (1999); J.M. Cline, C. Grojean, and G. Servant, Phys. Rev. Lett. 83, 4245 (1999); D.J. Chung and K. Freese, Phys. Rev. D 61, 023511 (2000); H.B. Kim and H.D. Kim, ibid. 61, 064003 (2000); P. Kanti, I.I. Kogan, K.A. Olive, and M. Pospelov, Phys. Lett. B 468, 31 (1999); J. Cline, C. Grojean, and G. Servant, ibid. 472, 302 (2000); P. Kraus, J. High Energy Phys. 12, 011 (1999); E.E. Flanagan, S.H. Tye, and I. Wasserman, Phys. Rev. D 62, 044039 (2000); C. Csaki, M. Graesser, L. Randall, and J. Terning, ibid. 62, 045015 (2000); D. Ida, J. High Energy Phys. 09, 014 (2000); M. Cvetic and J. Wang, Phys. Rev. D 61, 124020 (2000); P. Kanti, I.I. Kogan, K.A. Olive, and M. Pospelov, ibid. 61, 106004 (2000); S. Mukohyama, T. Shiromizu, and K. Maeda, ibid. 62, 024028 (2000); 63, 029901(E) (2000).

[18] R. Maartens, D. Wands, B.A. Bassett, and I. Heard, Phys. Rev. D 62, 041301 (2000); S. Nojiri and S.D. Odintsov, Phys. Lett. B 484, 119 (2000); J. High Energy Phys. 07, 049 (2000); S. Nojiri, O. Obregon, and S.D. Odintsov, Phys. Rev. D 62, 104003 (2000); T. Hertog and H.S. Reall, ibid. 63, 083504
(2001); S. Nojiri, S.D. Odintsov, and K.E. Osetrin, ibid. 63, 084016 (2001); R. Maartens, V. Sahni, and T.D. Saini, ibid. 63, 063509 (2001); R.M. Hawkins and J.E. Lidsey, ibid. 63, 041301 (2001); S. Kobayashi, K. Koyama, and J. Soda, Phys. Lett. B 501, 157 (2001).

[19] D. Tilley and R. Maartens, Class. Quantum Grav. 17, 287 (2000); E. Papantonopoulos and I. Pappa, Phys. Rev. D 63, 103506 (2001); S. Tsujikawa, K. Maeda, and S. Mizuno, ibid. 63, 123511 (2001); L. Anchordoqui and K. Olsen, Mod. Phys. Lett. A 16, 1157 (2001); K. Maeda, astro-ph/0012313; J. Yokoyama and Y. Himemoto, Phys. Rev. D (to be published), hep-ph/0103115; G. Huey and J.E. Lidsey, Phys. Lett. B 514, 217 (2001); B. Chen and F. Lin, hep-th/0106054.

[20] C. Barceló and M. Visser, Phys. Lett. B 482, 183 (2000); H. Stoica, S.H. Henry Tye, and I. Wasserman, ibid. 482, 205 (2000); R. Maartens, Phys. Rev. D 62, 084023 (2000); C. van de Bruck, M. Dorca, R.H. Brandenberger, and A. Lukas, ibid. 62, 123515 (2000); P. Bowcock, C. Charmousis, and R. Gregory, Class. Quantum Grav. 17, 4745 (2000); N.J. Kim, H.W. Lee, and Y.S. Myung, Phys. Lett. B 504, 323 (2001); A. Hebecker and J. March-Russell, Nucl. Phys. B608, 375 (2001); J. Khoury, B.A. Ovrut, P.J. Steinhardt, and N. Turok, hep-th/0103239; C.-M Chen, T. Harko, and M.K. Mak, Phys. Rev. D 64, 044013 (2001); Y.S. Myung, hep-th/0103241; S. Bhattacharya, D. Choudhury, D.P. Jatkar, and A.A. Sen, hep-th/0103248; D.H. Coule, gr-qc/0104016; N. Sago, Y. Himemoto, and M. Sasaki, gr-qc/0104033; N.J. Kim, H.W. Lee, Y.S. Myung, and G. Kang, Phys. Rev. D 64, 064022 (2001); R.-G. Cai, Y.S. Myung, and N. Ohta, hep-th/0105070; M. Ito, hep-th/0105186; R.-G. Cai and Y.-Z. Zhang, Phys. Rev. D (to be published), hep-th/0105214.

[21] H.A. Chamblin and H.S. Reall, Nucl. Phys. B562, 133 (1999); K. Enqvist, E. Keski-Vakkuri, and S. Rasanen, Phys. Rev. D 64, 044017 (2001); Y. Himemoto and M. Sasaki, ibid. 63, 044015 (2001); N. Sago, Y. Himemoto, and M. Sasaki, gr-qc/0104033.

[22] W. Israel, Nuovo Cimento B 44, 1 (1966); 48, 463(E) (1967). See also S.K. Blau, E.I. Guendelman, and A.H. Guth, Phys. Rev. D 35, 1747 (1987); D. Hochberg and T.W. Kephart, Phys. Rev. Lett. 70, 2665 (1993); M. Cvetic and H.H. Soleng, Phys. Rep. 282, 159 (1997); B. Carter, gr-qc/0012036; M. Visser, Lorentzian Wormholes (AIP Press, Woodbury, NY, 1995).

[23] G.T. Horowitz and D. Marolf, J. High Energy Phys. 07, 014 (1998).

[24] P. Horava and E. Witten, http://theory.theory.tifr.res.in/strings/ Proceedings/; R. Bousso, J. High Energy Phys. 04, 035 (2001); A. Chamblin and N.D. Lambert, Phys. Lett. B 508, 369 (2001); V. Balasubramanian, P. Horava, and D. Minic, J. High Energy Phys. 05, 043 (2001); E. Witten, hep-th/0106109.

[25] In more general cases one can take these two copies to be different. R.A. Battye and B. Carter, Phys. Lett. B 509, 331 (2001); R.A. Battye, B. Carter, A. Mennim, and J.-P Uzan, Phys. Rev. D (to be published), hep-th/0105091; B. Carter, J.-P. Uzan, R.A. Battye, and A. Mennim, gr-qc/0106038.

[26] See, e.g., W.S. Massey, Algebraic Topology (Springer, Berlin, 1977). The cut and paste discussed here results from the trivial homeomorphism, $h \equiv$ identity.

[27] G.W. Gibbons and S.W. Hawking, Phys. Rev. D 15, 2752 (1977). 
[28] D. Hochberg, C. Molina-Paris, and M. Visser, Phys. Rev. D 59, 044011 (1999).

[29] W.H. Press, S.A. Teukolsky, W.T. Vetterling, and B.P. Flannery, Numerical Recipes in Fortran (Cambridge University Press, Cambridge, England, 1993).

[30] J. Stoer and R. Bullirsch, Introduction to Numerical Analysis (Springer-Verlag, New York, 1991).

[31] R. Maartens, gr-qc/0101059.

[32] C. Barcelo and M. Visser, Phys. Rev. D 63, 024004 (2001); J. High Energy Phys. 10, 019 (2000).

[33] L.A. Anchordoqui and S.E. Perez Bergliaffa, Phys. Rev. D 62, 067502 (2000).

[34] J. Maldacena and C. Nuñez, Int. J. Mod. Phys. A 16, 822 (2001).

[35] S.B. Giddings, S. Kachru, and J. Polchinski, hep-th/0105097.

[36] C. Nuñez, I.Y. Park, M. Schvellinger, and T.A. Tran, J. High Energy Phys. 04, 025 (2001).

[37] M.J. Duff, J.T. Liu, and K.S. Stelle, hep-th/0007120; M. Cvetic, M.J. Duff, T.J. Liu, C.N. Pope, and K.S. Stelle, Nucl. Phys. B605, 141 (2001).

[38] J. Garriga and M. Sasaki, Phys. Rev. D 62, 043523 (2000); P.F. Gonzalez-Diaz, Phys. Lett. B 512, 127 (2001).

[39] J. Maldacena, Adv. Theor. Math. Phys. 2, 231 (1998) [Int. J. Theor. Phys. 38, 1113 (1998)].

[40] The prime example here being the duality between type IIB on $\mathrm{AdS}_{5} \times \mathrm{S}^{5}$ and $\mathcal{N}=4$ supersymmetric $U(N)$ Yang-Mills in $d$ $=4$ with coupling $g_{Y M}$ (the 't Hooft coupling is defined as $\lambda$ $\left.=g_{Y M}^{2} N\right)$. See [39].

[41] E. Witten, Adv. Theor. Math. Phys. 2, 253 (1998); S.S. Gubser, I.R. Klebanov, and A.M. Polyakov, Phys. Lett. B 428, 105 (1998).

[42] A. Strominger and C. Vafa, Phys. Lett. B 379, 99 (1996); J.M. Maldacena, Ph.D. thesis, Princeton University, 1996, hep-th/9607235.

[43] S.S. Gubser, Phys. Rev. D 63, 084017 (2001); S. Nojiri, S.D. Odintsov, and S. Zerbini, ibid. 62, 064006 (2000); S.W. Hawking, T. Hertog, and H.S. Reall, ibid. 62, 043501 (2000); L. Anchordoqui, C. Nuñez, and K. Olsen, J. High Energy Phys. 10, 050 (2000).

[44] B.T. McInnes, Nucl. Phys. B602, 132 (2001); N.S. Deger and A. Kaya, J. High Energy Phys. 05, 030 (2001); S. de Haro, K.
Skenderis, and S.N. Solodukhin, hep-th/0011230; K. Koyama and J. Soda, J. High Energy Phys. 05, 027 (2001); I. Savonije and E. Verlinde, Phys. Lett. B 507, 305 (2001); T. Shiromizu and D. Ida, Phys. Rev. D 64, 044015 (2001); K. Ghoroku and A. Nakamura, ibid. (to be published), hep-th/0103071; S. Nojiri and S.D. Odintsov, hep-th/0103078; M. Mintchev, hep-th/0103259; M. Perez-Victoria, J. High Energy Phys. 05, 064 (2001); S. Nojiri, S.D. Odintsov, and S. Ogushi, hep-th/0105117; T. Shiromizu, T. Torii, and D. Ida, hep-th/0105256; I.Z. Rothstein, Phys. Rev. D (to be published), hep-th/0106022.

[45] Within this framework the universe can be thought of as a compactification of $\mathrm{M}$ theory on $\mathrm{AdS}_{5}$. There are several compactifications of string/M theory that satisfy this condition. Apart from the very well known compactifications of II B on $\mathrm{AdS}_{5} \times \mathrm{S}^{5} / \Gamma$, we can mention the ones studied in A. Fayyazuddin and D.J. Smith, J. High Energy Phys. 10, 023 (2000). See also [34].

[46] J.M. Maldacena, hep-th/0106112.

[47] O. Aharony, S.S. Gubser, J. Maldacena, H. Ooguri, and Y. Oz, Phys. Rep. 323, 183 (2000).

[48] S.W. Hawking and G.F.R. Ellis, The Large Scale Structure of the Space-time (Cambridge University Press, Cambridge, England, 1973).

[49] Quotients between geometries are also used to describe black holes (like in the case of $\mathrm{AdS}_{3}$ with the BTZ black hole). See M. Bañados, C. Teitelboim, and J. Zanelli, Phys. Rev. Lett. 69, 1849 (1992); M. Bañados, M. Henneaux, C. Teitelboim, and J. Zanelli, Phys. Rev. D 48, 1506 (1993).

[50] Notice that the influence of the KK modes should be important near the big-bang/big-crunch, but more or less negligible in the middle of the evolution.

[51] Although $\mathrm{H}^{2}$ is not a compact space, the low energy degrees of freedom cannot be excited on this manifold because they have much larger wavelengths when compared to the characteristic length of $\mathrm{H}^{2}$.

[52] D. Marolf (private communication).

[53] G. Dvali, G. Gabadadze, and M. Porrati, Phys. Lett. B 485, 208 (2000); G. Dvali and G. Gabadadze, Phys. Rev. D 63, 065007 (2001); see also Dvali-Gabadadze-Kolanovic-Nitti in Ref. [14]. 